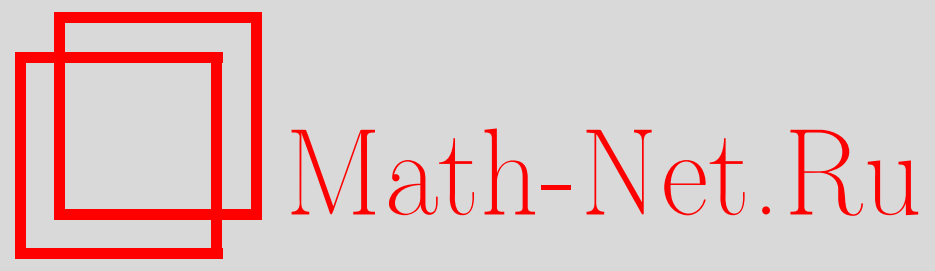

И. А. Чеплюкова, Возникновение гигантского дерева в случайном лесе, Дискрет. матем., 1998, том 10, выпуск 1, 111-126

DOI: https://doi.org/10.4213/dm408

Использование Общероссийского математического портала Math-Net.Ru подразумевает, что вы прочитали и согласны с пользовательским соглашением http://www . mathnet.ru/rus/agreement

Параметры загрузки:

IP : 54.224 .60 .19

26 апреля 2023 г., 13:27:10 


\title{
Возникновение гигантского дерева в случайном лесе
}

\author{
(С) 1998 г. И. А. Чеплюкова
}

Рассматриваются случайные леса, состоящие из $N$ деревьев просто генерируемого семейства корневых деревьев с $n$ некорневыми вершинами. Для таких лесов проведены исследования условий возникновения гигантского дерева в случайном лесе и показано, что гигантская компонента появляется только тогда, когда $N, n \rightarrow \infty$ так, что $n / N^{2} \rightarrow \infty$.

Работа выполнена при поддержке Российского фонда фундаментальных исследований, проект 97-01-00065.

В [1] было введено понятие просто генерируемого семейства $\mathscr{F}$ корневых деревьев. В соответствии с этим понятием существует степенной ряд

$$
F(z)=\sum_{k=0}^{\infty} p_{k} z^{k}
$$

где $p_{0}>0, p_{k} \geqslant 0$ при $k \geqslant 1$, коэффициенты которого определяют вес $\omega(T)$ плоского дерева $T$ с висячим корнем по формуле

$$
\omega(T)=\prod_{k \geqslant 0} p_{k}^{m_{k}}
$$

в которой $m_{k}$ означает число вершин дерева кратности $k$ (кратность вершины есть число дуг, выходящих из нее при ориентации дуг от корня). Пусть $\mathscr{F}_{n}-$ множество деревьев $T \in \mathscr{F}$, содержащих $n$ вершин. Согласно [1] семейство $\mathscr{F}_{n}$ есть просто генерируемое, если производящая функция

$$
\varphi(z)=\sum_{n=0}^{\infty} y_{n} z^{n},
$$

где

$$
y_{n}=\sum_{T \in \mathscr{F}_{n}} \omega(T)
$$

удовлетворяет уравнению

$$
\varphi(z)=z F(\varphi(z))
$$

Простейшими примерами просто генерируемых деревьев являются плоские деревья с висячими корнями, деревья с помеченными вершинами, бинарные деревья. 
Если $F(1)=1$, то семейство просто генерируемых деревьев можно интерпретировать $[2,3]$ как совокупность реализаций ветвящегося процесса Гальтона-Ватсона с производящей функцией (1) распределения числа прямых потомков каждой частицы. При этом вес $\omega(T)$ равен вероятности реализации $T$, а $y_{n}$ есть вероятность того, что в ветвящемся процессе существовало $n$ частиц за все время его эволюции. Известно (см., например, [4]), что для таких процессов соотношение (2) выполняется.

В настоящей статье рассматриваются леса, состоящие из $N$ деревьев из $\mathscr{F}$. Множество таких лесов в соответствии со сказанным выше естественно рассматривать как ветвящийся процесс Гальтона-Ватсона $G$, начинающийся с $N$ частиц. Обозначим $\mathscr{F}_{N, n}$ совокупность рассматриваемых лесов с $n$ некорневыми вершинами. Ветвящийся процесс $G$ естественным образом индуцирует распределение вероятностей на $\mathscr{F}_{N, n}$, при этом, если $G_{N, n}$ - реализация процесса $G$ с $N+n$ частицами, To

$$
\mathbf{P}\left\{G=G_{N, n} \mid \nu=N+n\right\}=\frac{p_{0}^{m_{0}} p_{1}^{m_{1}} \ldots p_{n}^{m_{n}}}{\mathbf{P}\{\nu=N+n\}},
$$

где $\nu$ - общее число частиц, существовавших в ветвящемся процессе,

$$
m_{0}+m_{1}+\ldots+m_{n}=N+n, \quad m_{1}+2 m_{2}+\ldots+n m_{n}=n
$$

и $p_{i}$, в соответствии с (1), есть вероятность рождения $i$ прямых потомков у одной частицы, $i=0,1,2, \ldots$

В [5] получены предельные распределения важнейших характеристик случайных лесов. Заметим, что рассматриваемый здесь класс лесов $\mathscr{F}_{N, n}$ шире рассматриваемого в [5], поскольку там предполагается, что

$$
\mathbf{P}\left\{G=G_{N, n} \mid \nu=N+n\right\}=\frac{\left|\Delta\left(G_{N, n}\right)\right|}{\left|\mathscr{F}_{N, n}\right|},
$$

где $\Delta\left(G_{N, n}\right)$ означает класс лесов из $\mathscr{F}_{N, n}$ изоморфных реализации $G_{N, n}$, a $\left|\Delta\left(G_{N, n}\right)\right|$ и $\left|\mathscr{F}_{N, n}\right|$ - мощности множеств $\Delta\left(G_{N, n}\right)$ и $\mathscr{F}_{N, n}$ соответственно.

Пусть $\xi\left(G_{N, n}\right)$ - некоторая числовая характеристика $G_{N, n}$, а $\xi(G)$ - аналогичная характеристика соответствующего ветвящегося процесса $G$. Если лес $f$, принадлежащий $\mathscr{F}_{N, n}$, содержится в множестве $\Delta\left(G_{N, n}\right)$, то положим

$$
\xi=\xi(f)=\xi\left(G_{N, n}\right) .
$$

Лемма 1. Справедливо равенство

$$
\mathbf{P}\{\xi=x\}=\mathbf{P}\{\xi(G)=x \mid \nu=N+n\} .
$$

Доказательство. Пусть $\chi(A)$ - индикатор события $A$, а $\mathbf{P}\{f\}$ - вероятность появления леса $f$. Тогда

$$
\begin{aligned}
\mathbf{P}\{\xi=x\} & =\sum_{G_{N, n}} \sum_{f \in \Delta\left(G_{N, n}\right)} \mathbf{P}\{f\} \chi(\xi(f)=x) \\
& =\sum_{G_{N, n}} \chi\left(\xi\left(G_{N, n}\right)=x\right) \sum_{f \in \Delta\left(G_{N, n}\right)} \mathbf{P}\{f\} \\
& =\sum_{G_{N, n}} \chi\left(\xi\left(G_{N, n}\right)=x\right) \mathbf{P}\left\{G=G_{N, n} \mid \nu=N+n\right\} .
\end{aligned}
$$


Отсюда и из (3) следует утверждение леммы 1.

Эта лемма показывает, что изучение числовых характеристик случайного леса можно свести к исследованию аналогичных характеристик ветвящихся процессов при условии $\nu=N+n$. Лемма 1 является обобщением леммы 1.4.1 книги [5], поэтому все результаты [5], опирающиеся на эту лемму, оказываются справедливыми и для лесов из рассматриваемого множества $\mathscr{F}_{N, n}$. В [5], в частности, получены предельные распределения максимального объема дерева в случайном лесе при $N, n \rightarrow \infty$. По аналогии с эволюцией случайного графа говорят, что при некотором соотношении между параметрами $N, n \rightarrow \infty$, в случайном лесе возникает гигантское дерево, если число вершин в этом дереве имеет порядок $n$, в то время как каждое из остальных деревьев содержит меньшее по порядку число вершин. В [5] доказано, что число вершин в максимальном дереве имеет порядок $n$ в следующих двух случаях.

Пусть $\nu_{1}(\mathscr{F}), \ldots, \nu_{N}(\mathscr{F})$ - случайные величины, равные объемам деревьев из $\mathscr{F}_{N, n}$, имеющих корневые вершины с номерами $1, \ldots, N$ соответственно, а $\nu_{(1)}(\mathscr{F}), \ldots, \nu_{(N)}(\mathscr{F})$ - вариационный ряд объемов деревьев в лесе, полученный расположечием $\nu_{1}(\mathscr{F}), \ldots, \nu_{N}(\mathscr{F})$ в неубывающем порядке. Введем вспомогательную случайную величину $\eta$, имеющую целочисленное распределение

$$
\mathbf{P}\{\eta=k\}=p_{k}, \quad k=0,1,2, \ldots,
$$

с махсимальным шагом $d$ и производящей функцией (1), где множество значений $\eta$, имеющих ненулевую вероятность, содержит нуль и не совпадает с множеством $\{0,1\}$. Пусть

$$
\mathbf{M} \eta=1, \quad \mathbf{D} \eta=B, \quad F^{\prime \prime \prime}(1)<\infty
$$

а число прямых потомков одной частицы ветвящегося процесса $G$ имеет распределение

$$
p_{k}(\lambda)=\lambda^{k} p_{k} / F(\lambda), \quad k=0,1,2, \ldots
$$

где $0<\lambda \leqslant 1$.

В [5] доказаны следующие утверждения.

Теорема 1. Пусть $N, n \rightarrow \infty$ так, что $n$ пробегает значения, кратнъе $d, u$ $\mathrm{Bn} / \mathrm{N}^{2} \rightarrow \gamma$, где $\gamma$ - некоторая положительная постоянная.

Тогда для любого фиксированного положительного $z$

$$
\mathbf{P}\left\{\nu_{(N)}(\mathscr{F}) / n \leqslant z\right\} \rightarrow \exp \left\{\frac{1}{2 \gamma}\right\} \gamma^{3 / 2} \sum_{k=0}^{\infty} \frac{(-1)^{k}}{k !} I_{k}(\gamma z, \gamma)
$$

əде

$$
\begin{aligned}
I_{k}(u, v) & =\int_{X_{k}(u, v)} \frac{\exp \left\{-1 /\left(2\left(v-x_{1}-\ldots-x_{k}\right)\right)\right\} d x_{1} \ldots d x_{k}}{(2 \pi)^{k / 2}\left(x_{1} \ldots x_{k}\left(v-x_{1}-\ldots-x_{k}\right)\right)^{3 / 2}} \\
I_{0}(u, v) & =1 / \sqrt{2 v^{3} \exp \{1 / v\}} \\
X_{k}(u, v) & =\left\{x_{i} \geqslant u, i=1, \ldots, k, x_{1}+\ldots+x_{k} \leqslant v\right\}
\end{aligned}
$$


Теорема 2. Пусть $n, N \rightarrow \infty$ так, что $n$ пробегает значения, кратные $d, u$ $n / N^{2} \rightarrow \infty$. Тогда для любого фиксированного положительного $z$

$$
\mathbf{P}\left\{\left(n-\nu_{(N)}\right) B / N^{2} \leqslant z\right\} \rightarrow \frac{1}{\sqrt{2 \pi}} \int_{0}^{z} y^{-3 / 2} \exp \{-1 /(2 y)\} d y .
$$

Из этих теорем не видно, является ли в рассмотренных случаях максимальное дерево гигантским, поскольку не выяснено поведение следующего по величине члена вариационного ряда. В настоящей статье изучается поведение членов вариационного ряда $\nu_{(N-j)}(\mathscr{F})$ при любом фиксированном $j=0,1,2 \ldots$ Оказывается, что гигантское дерево возникает лишь в случае, когда $n, N \rightarrow \infty$ так, что $n / N^{2} \rightarrow \infty$.

Возникновение гигантского дерева в эволюции случайного леса из некорневых деревьев изучалось в $[6,7]$.

Ветвящийся процесс: $G$ распадается на $N$ процессов $G^{(1)}, \ldots, G^{(N)}$, каждый из которых начинается с одной частицы. Введем случайные величины $\nu^{(1)}, \ldots, \nu^{(N)}$, равные числу частиц, существовавших соответственно в процессах $G^{(1)}, \ldots, G^{(N)}$ до их вырождения.

В данной статье получены следующие результаты.

Теорема 3. Пусть $n, N \rightarrow \infty$ так, что $n$ пробегает значения, кратные $d$, $n / N \rightarrow 0$, а параметр $\lambda=\lambda(N, n)$ определяется соотношением

$$
\frac{\lambda F^{\prime}(\lambda)}{F(\lambda)}=\frac{n}{N+n}
$$

Пусть

$$
N \mathbf{P}\left\{\nu^{(1)}=r+1\right\} \rightarrow \infty, \quad N \mathbf{P}\left\{\nu^{(1)}=r+s+1\right\} \rightarrow \gamma,
$$

где $\gamma$ - неотрицательная постоянная, а натуралъные $r$ и $s$ кратны $d$ и удовлетворяют одному из условий:

(1) $r \rightarrow \infty, s=d$;

(2) $r$ фиксировано, $r \geqslant m+1$,

$$
\mathbf{P}\left\{\nu^{(1)}=r+s+1\right\}>0, \quad \mathbf{P}\left\{\nu^{(1)}=r+i+1\right\}=0,
$$

где $m$ - наименъиее челое положителъное число такое, что $p_{m}>0 u 0<$ $i<s$.

Тогда для любого фиксированного $j=0,1,2, \ldots$

$$
\begin{gathered}
\mathbf{P}\left\{\nu_{(N-j)}(\mathscr{F})=r+1\right\}=e^{-\gamma} \sum_{i=0}^{j} \frac{\gamma^{i}}{i !}, \\
\mathbf{P}\left\{\nu_{(N-j)}^{\prime}(\mathscr{F})=r+s+1\right\}=1-e^{-\gamma} \sum_{i=0}^{j} \frac{\gamma^{i}}{i !} .
\end{gathered}
$$

Теорема 4. Пусть $n, N \rightarrow \infty$ так, что $n$ пробегает значения, кратные $d, u$ $n / N \rightarrow b$, где $b-$ положительная постоянная. Пусть параметр $\lambda=\lambda(N, n)$ определяется соотношением (4),

$$
\alpha=\left(\lambda_{b} / F\left(\lambda_{b}\right)\right)^{d},
$$


где $\lambda_{b}-$ коренъ уравнения

$$
\lambda F^{\prime}(\lambda) / F(\lambda)=b /(b+1)
$$

Eсли $r=r(N, n)$ пробегает значения, кратные $d$, такие, что

$$
\frac{N}{F(\lambda)}\left(\frac{\lambda}{F(\lambda)}\right)^{r} \frac{d}{r^{3 / 2} \sqrt{2 \pi B}} \rightarrow \gamma
$$

где $\gamma$ - положителъная постоянная, то для любых фиксированных $k=0, \pm 1, \pm 2, \ldots$ $u j=0,1,2, \ldots$

$$
\mathbf{P}\left\{\nu_{(N-j)}(\mathscr{F}) \leqslant r+k d+1\right\} \rightarrow \exp \left\{-\frac{\gamma \alpha^{k+1}}{1-\alpha}\right\} \sum_{i=0}^{j} \frac{1}{i !}\left(\frac{\gamma \alpha^{k+1}}{1-\alpha}\right)^{i}
$$

Теорема 5. Пусть $n, N \rightarrow \infty$ так, что $n$ пробегает значения, кратные $d, u$ $n / N \rightarrow \infty, n / N^{2} \rightarrow 0$. Пусть параметр $\lambda=\lambda(N, n)$ определяется соотношением (4). Тогда для любого фиксированного $z$

$$
\mathbf{P}\left\{\dot{\beta \nu_{(N-j)}}(\mathscr{F})-u \leqslant z\right\} \rightarrow e^{-e^{-z}} \sum_{i=0}^{j} \frac{e^{-i z}}{i !}
$$

гдe

$$
\beta=\beta(\lambda)=-\ln (\lambda / F(\lambda)),
$$

а $u=u(\lambda)$ выбрано так, что

$$
N \beta^{1 / 2} u^{-3 / 2} e^{-u}=\sqrt{2 \pi B}
$$

Теорема 6. Пусть $n, N \rightarrow \infty$ так, что $n$ пробегает значения, кратные $d, u$ Bn $/ N^{2} \rightarrow \gamma$, где $\gamma-$ положстельная постоянная. Тогда для любого фиксированного положительного $z$ и любого фихсированного $j=0,1,2, \ldots$

$$
\mathbf{P}\left\{\nu_{(N-j)}(\mathscr{F}) / n \leqslant z\right\} \rightarrow \exp \left\{\frac{1}{2 \gamma}\right\} \gamma^{3 / 2} \sum_{k=0}^{\infty} \frac{(-1)^{k}}{k !} \sum_{i=0}^{j} \frac{1}{i !} I_{k+i}(\gamma z, \gamma)
$$

$2 \partial e$

$$
\begin{aligned}
I_{k}(u, v) & =\int_{X_{k}(u, v)} \frac{\exp \left\{-1 /\left(2\left(v-x_{1}-\ldots-x_{k}\right)\right)\right\} d x_{1} \ldots d x_{k}}{(2 \pi)^{k / 2}\left(x_{1} \ldots x_{k}\left(v-x_{1}-\ldots-x_{k}\right)\right)^{3 / 2}} \\
I_{0}(u, v) & =1 / \sqrt{2 v^{3} \exp \{1 / v\}} \\
X_{k}(u, v) & =\left\{x_{i} \geqslant u, i=1, \ldots, k, x_{1}+\ldots+x_{k} \leqslant v\right\}, \quad k=1,2, \ldots
\end{aligned}
$$

Теорема 7. Пусть $n, N \rightarrow \infty$ так, что $n$ пробегает значения, кратные $d, u$ $n / N^{2} \rightarrow \infty$. Тогда для любого фиксированного положителъного $z$

$$
\mathbf{P}\left\{\nu_{(N-1)}(\mathscr{F}) \leqslant z N^{2}\right\} \rightarrow e^{-E(0, z)},
$$

əวe

$$
E(0, z)=\sqrt{2 /(z \pi B)} .
$$


Из теорем 1-7 видно, что гигантское дерево возникает в случайном лесе только в случае, когда $n, N \rightarrow \infty$ так, что $n / N^{2} \rightarrow \infty$.

Для доказательства теорем 2-7 докажем несколько лемм.

Из леммы 1 следует, что для всех $n$, удовлетворяющих условию $\mathbf{P}\{\nu=N+n\}>0$, справедливо соотношение

$$
\mathbf{P}\left\{\nu_{1}(\mathscr{F})=k_{1}, \ldots, \nu_{N}(\mathscr{F})=k_{N}\right\}=\mathbf{P}\left\{\nu^{(1)}=k_{1}, \ldots, \nu^{(N)}=\dot{k}_{N} \mid \nu=N+n\right\} .
$$

Введем вспомогательные независимые одинаково распределенные случайные величины $\nu_{r}^{(1)}, \ldots, \nu_{r}^{(N)}$ и $\tilde{\nu}_{r}^{(1)}, \ldots, \tilde{\nu}_{r}^{(N)}$ для которых

$$
\begin{aligned}
& \mathbf{P}\left\{\nu_{r}^{(i)}=k\right\}=\mathbf{P}\left\{\nu^{(i)}=k \mid \nu^{(i)} \leqslant r+1\right\}, \\
& \mathbf{P}\left\{\tilde{\nu}_{r}^{(i)}=k\right\}=\mathbf{P}\left\{\nu^{(i)}=k \mid \nu^{(i)}>r+1\right\}, \quad i=1, \ldots, N, \quad k=1,2, \ldots
\end{aligned}
$$

Обозначим также

$$
\begin{gathered}
\nu_{r, i}=\nu_{r}^{(1)}+\ldots+\nu_{r}^{(N-i)}+\tilde{\nu}_{r}^{(N-i+1)}+\ldots+\tilde{\nu}_{r}^{(N)}, \quad i=0,1, \ldots, N-1, \\
P_{r}=\mathbf{P}\left\{\nu^{(1)}>r+1\right\}, \quad \sigma^{2}=\mathbf{D} \nu^{(1)} .
\end{gathered}
$$

Тогда

$$
P_{r}=\sum_{k=1}^{\infty} \mathbf{P}\left\{\nu^{(1)}=r+k d+1\right\}
$$

Из (7) и соотношения

$$
\begin{array}{r}
\mathbf{P}\left\{\nu_{(N-j)}(\mathscr{F}) \leqslant r+1\right\}=\sum_{i=0}^{j}\left(\begin{array}{c}
N \\
i
\end{array}\right) \mathbf{P}\left\{\nu_{(1)}(\mathscr{F}) \leqslant r+1, \ldots, \nu_{(N-i)}(\mathscr{F}) \leqslant r+1,\right. \\
\left.\nu_{(N-i+1)}(\mathscr{F})>r+1, \ldots, \nu_{(N)}(\mathscr{F})>r+1\right\}
\end{array}
$$

нетрудно получить следующий результат, являющийся обобщением леммы 1.2 .2 из [5].

Лемма 2. При $n$ maкux, чmo $\mathbf{P}\{\nu=N+n\}>0$,

$$
\begin{aligned}
\mathbf{P}\left\{\nu_{(N-j)}(\mathscr{F}) \leqslant r+1\right\} & \\
& =\sum_{i=0}^{j}\left(1-P_{r}\right)^{N-i} P_{r}^{i} \frac{N(N-1) \ldots(N-i+1)}{i !} \frac{\mathbf{P}\left\{\nu_{r, i}=n+N\right\}}{\mathbf{P}\{\nu=n+N\}} .
\end{aligned}
$$

Лемма 2 показывает, что для получения предельных распределений случайных величин $\nu_{(N-j)}(\mathscr{F})$ достаточно изучить асимптотику $\mathbf{P}\left\{\nu_{r, i}=N+n\right\}$ и поведение $N P_{r}$ в случае $n / N^{2} \rightarrow \infty$, поскольку асимптотическое поведение бинома $\left(1-P_{r}\right)^{N-i}$ в остальных случаях и вероятностей $P_{r}$ и $\mathbf{P}\{\nu=n+N\}$ изучено в [5]. Ниже, в леммах 3-8, описывается предельное поведение $\mathbf{P}\left\{\nu_{r, i}=N+n\right\}$ и $N P_{r}$, а затем с помощью этих результатов доказываются утверждения теорем 3-7.

Пусть $\varphi_{r, t}(u)$ означает характеристическую функцию случайной величины

$$
\frac{\nu_{r, t}-N-n}{\sigma \sqrt{N}}, \quad t=1, \ldots, j .
$$

Справедливо следующее утверждение. 
Лемма 3. Пусть $n, N \rightarrow \infty, n / N^{2} \rightarrow 0$, параметр $\lambda=\lambda(N, n)$ определяется соотношением (4), а $r=r(N, n)$ пробегает значения, кратные $d$, тахие, что $N P_{r} \rightarrow \gamma$, где $\gamma$ - положительная постоянная.

Тогда для любого фиксированного $u$

$$
\varphi_{r, t}(u) \rightarrow \exp \left\{-u^{2} / 2\right\}
$$

Доказательство. Лемма 3 доказывается аналогично лемме 2.4 .1 из [5]. Обозначим через $\varphi_{\tilde{\nu}_{r}^{(1)}}(u), \varphi_{\nu_{r}^{(1)}}(u), \varphi(u)$ характеристические функции случайных величин $\tilde{\nu}_{r}^{(1)}$, $\nu_{r}^{(1)}, \nu^{(1)}$ соответственно. Нетрудно получить, что

$$
\begin{aligned}
& \varphi_{\tilde{\nu}_{r}^{(1)}}(u)=P_{r}^{-1} \sum_{k=1}^{\infty} \mathbf{P}\left\{\nu^{(1)}=r+k d+1\right\} \exp \{i u(r+k d+1)\}, \\
& \varphi_{\nu_{r}^{(1)}}(u)=\left(1-P_{r}\right)^{-1}\left(\varphi(u)-\sum_{k=1}^{\infty} \mathbf{P}\left\{\nu^{(1)}=r+k d+1\right\} \exp \{i u(r+k d+1)\}\right) .
\end{aligned}
$$

Тогда

$$
\begin{aligned}
\varphi_{r, t}(u)= & \frac{1}{\left(1-P_{r}\right)^{N-t}}\left(\varphi\left(\frac{u}{\sigma \sqrt{N}}\right)-\sum_{k=1}^{\infty} \mathbf{P}\left\{\nu^{(1)}=r+k d+1\right\} \exp \left\{i u \frac{r+k d+1}{\sigma \sqrt{N}}\right\}\right)^{N-t} \\
& \times P_{r}^{-t}\left(\sum_{k=1}^{\infty} \mathbf{P}\left\{\nu^{(1)}=r+k d+1\right\} \exp \{i u(r+k d+1) /(\sigma \sqrt{N})\}\right)^{t} \\
& \times \exp \{-i u(N+n) /(\sigma \sqrt{N})\} .
\end{aligned}
$$

Согласно лемме 2.4.1 из [5] справедливо соотношение

$$
\begin{aligned}
\varphi_{r, t}(u)=(1+o(1)) & e^{-u^{2} / 2} P_{r}^{-t} \\
& \times\left(\sum_{k=1}^{\infty} \mathrm{P}\left\{\nu^{(1)}=r+k d+1\right\} \exp \{i u(r+k d+1) /(\sigma \sqrt{N})\}\right)^{t} .
\end{aligned}
$$

Нетрудно показать, что

$$
\sum_{k=1}^{\infty} \mathbf{P}\left\{\nu^{(1)}=r+k d+1\right\} \exp \{i u(r+k d+1) /(\sigma \sqrt{N})\}=P_{r}+Q(u),
$$

где

$$
|Q(u)| \leqslant|u|(\sigma \sqrt{N})^{-1} \sum_{k=1}^{\infty}(r+k d+1) \mathbf{P}\left\{\nu^{(1)}=r+k d+1\right\} .
$$

В [5] показано, что

$$
(\sigma \sqrt{N})^{-1} \sum_{k=1}^{\infty}(r+k d+1) \mathbf{P}\left\{\nu^{(1)}=r+k d+1\right\}=o\left(N^{-1}\right)
$$

Из (8)-(11) следует утверждение леммы 3. 
Лемма 3 утверждает, что распределение случайной величины $\nu_{r, t}$ слабо сходится к нормальному закону с параметрами $(N+n, \sigma \sqrt{N})$. Докажем локальное сближение этих распределений.

Лемма 4. Пусть выполнены условия леммъ 3 и, кроме того, пусть $N \lambda^{m+l} \rightarrow \infty$, где $l$ - натуральное число, не кратное $m$, удовлетворлющее условию $p_{m+l}>0$, если такого $l$ нет, mo $l=0$.

Тогда для любого фиксированного $t=0,1, \ldots, j$ и для челых неотричательных $h$, кратных $d$, равномерно относительно $(h-n) /(\sigma \sqrt{N})$ в любом конечном интервале

$$
\mathbf{P}\left\{\nu_{r, t}=N+h\right\}=\frac{d(1+o(1))}{\sigma \sqrt{2 \pi N}} \exp \left\{-\frac{(h-n)^{2}}{2 \sigma^{2} N}\right\} .
$$

Доказательство. Используя формулу обращения, представим $\mathbf{P}\left\{\nu_{r, t}=N+h\right\}$ в виде интеграла

$$
\mathbf{P}\left\{\nu_{r, t}=N+h\right\}=\frac{d}{2 \pi \sigma \sqrt{N}} \int_{-\pi \sigma \sqrt{N} / d}^{\pi \sigma \sqrt{N} / d} \exp \{-i u z\} \varphi_{r, t}(u) d u,
$$

где $z=(h-n) /(\sigma \sqrt{N})$. Рассмотрим разность

$$
R=2 \pi\left(\frac{\sigma \sqrt{N}}{d} \mathbf{P}\left\{\nu_{r, t}=N+h\right\}-\frac{1}{\sqrt{2 \pi}} e^{-z^{2} / 2}\right) .
$$

Поскольку

$$
\frac{1}{\sqrt{2 \pi}} e^{-z^{2} / 2}=\frac{1}{2 \pi} \int_{-\infty}^{\infty} e^{-i x z-x^{2} / 2} d x,
$$

разность $R$ можно представить в виде суммы четырех интегралов:

$$
R=I_{1}+I_{2}+I_{3}+I_{4}
$$

где

$$
\begin{aligned}
& I_{1}=\int_{-A}^{A} e^{-i u z}\left(\varphi_{r, t}(u)-e^{-u^{2} / 2}\right) d u, \\
& I_{2}=\int_{A<|u| \leqslant \varepsilon \sigma \sqrt{N}} e^{-i u z} \varphi_{r, t}(u) d u, \\
& I_{3}=\int_{\varepsilon \sigma \sqrt{N}<|u| \leqslant d^{-1} \pi \sigma \sqrt{N}} e^{-i u z} \varphi_{r, t}(u) d u, \\
& I_{4}=-\int_{A<|u|} e^{-i u z-u^{2} / 2} d u,
\end{aligned}
$$

$A$ и $\varepsilon-$ положительные постоянные. В силу леммы 3 интеграл $I_{1} \rightarrow 0$. Оценки интегралов $I_{2}, I_{3}, I_{4}$ аналогичны соответствующим оценкам, приведенным при доказательстве леммы 2.4.2 в книге [5], где показано, что выбором достаточно больших $N, n, A$ и достаточно малого $\varepsilon$ интегралы $I_{2}, I_{3}, I_{4}$ можно сделать сколь угодно малыми, что и доказывает лемму 4. 
Лемма 5. Пусть $n, N \rightarrow \infty, B n / N^{2} \rightarrow \gamma$, где $\gamma-$ положителъная постоянная. Пустъ $v=(N+h) B / N^{2}, h$ - натуральное число, кратное $d$. Если $r$ кратно

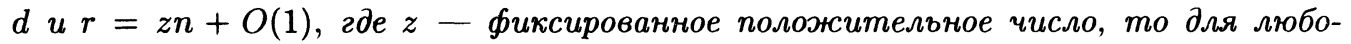
го фиксированного натуралъного $t$ равномерно относителъно $v$ в любом конечном интервале вида $0<v_{0} \leqslant v \leqslant v_{1}$

$$
\frac{N^{2}}{B} \mathbf{P}\left\{B \nu_{r, t} / N^{2}=v\right\} \rightarrow \frac{d e^{E(0, z)}}{\sqrt{2 \pi} E^{t}(0, z)} \sum_{k=0}^{\infty} \frac{(-1)^{k}}{k !} I_{k+t}(\gamma z, v),
$$

где $E(0, z)$ и $I_{k}(\gamma z, v)$ определенъ соотношениями (6) $u$ (5) соответственно.

Доказателъство. Обозначим через $\psi_{r, t}(u)$ характеристическую функцию случайной величины $B \nu_{r, t} / N^{2}$. Тогда

$$
\begin{gathered}
\psi_{r, t}(u)=\frac{1}{\left(1-P_{r}\right)^{N-t}}\left(\varphi\left(B u / N^{2}\right)-\sum_{k=1}^{\infty} \mathbf{P}\left\{\nu^{(1)}=r+k d+1\right\} \exp \left\{i u B(r+k d+1) / N^{2}\right\}\right)^{N-t} \\
\times P_{r}^{-t}\left(\sum_{k=1}^{\infty} \mathbf{P}\left\{\nu^{(1)}=r+k d+1\right\} \exp \left\{i u B(r+k d+1) / N^{2}\right\}\right)^{t}
\end{gathered}
$$

В [5] показаңо, что

$$
\varphi^{N}\left(u B / N^{2}\right) \rightarrow \exp \{-\sqrt{-2 i u}\}
$$

а из леммы 1.3 .12 из [5] следует, что если $N, n \rightarrow \infty$ так, что $B u / N^{2} \rightarrow \gamma$, то

$$
\begin{array}{r}
\sum_{k=1}^{\infty} \mathbf{P}\left\{\nu^{(1)}=r+k d+1\right\} \exp \left\{i u B(r+k d+1) / N^{2}\right\}=\frac{E(u, v)}{N}(1+o(1)), \\
\sum_{k=1}^{\infty} \mathbf{P}\left\{\nu^{(1)}=r+k d+1\right\}=\frac{E(0, z)}{N}(1+o(1)),
\end{array}
$$

где

$$
E(u, z)=\frac{1}{\sqrt{2 \pi}} \int_{\gamma z}^{\infty} y^{-3 / 2} e^{i u y} d y
$$

Тогда

$$
\psi_{r, t}(u)=\exp \{-\sqrt{-2 i u}-E(u, z)+E(0, z)\} \frac{E^{t}(u, z)}{E^{t}(0, z)}(1+o(1)) .
$$

Поскольку $E(u, z)$ является преобразованием Фурье функции

$$
f(t)= \begin{cases}\left(\sqrt{2 \pi t^{3}}\right)^{-1}, & t \geqslant \gamma z \\ 0, & t<\gamma z\end{cases}
$$

и $\exp \{-\sqrt{-2 i u}\}-$ характеристическая функция устойчивого закона с показателем $\alpha=1 / 2$, имеющего плотность

$$
\frac{1}{\sqrt{2 \pi}} x^{-3 / 2} e^{-1 /(2 x)}, \quad x>0
$$


нетрудно показать, что $\psi_{r, t}(u)$ сходится к характеристической функции распределения с плотностью

$$
g(v)=\frac{e^{E(0, z)}}{\sqrt{2 \pi} E^{t}(0, z)} \sum_{k=0}^{\infty} \frac{(-1)^{k}}{k !} I_{k}(\gamma z, v) .
$$

По формуле обращения

$$
\mathbf{P}\left\{\nu_{r, t}==N+h\right\}=\frac{d B}{2 \pi N^{2}} \int_{-N^{2} \pi /(d B)}^{N^{2} \pi /(d B)} e^{-i t v} \psi_{r, t}(t) d t .
$$

Поэтому

$$
\frac{2 \pi N^{2}}{d B} \mathbf{P}\left\{B \nu_{r, t} / N^{2}=v\right\}-2 \pi g(v)=I_{1}+I_{2}+I_{3}+I_{4}
$$

где

$$
\begin{aligned}
& I_{1}=\int_{-A}^{A} e^{-i u v}\left(\psi_{r, t}(u)-\exp \{-\sqrt{-2 i u}-E(u, z)+E(0, z)\} \frac{E^{t}(u, z)}{E^{t}(0, z)}\right) d u, \\
& I_{2}=\int_{A<|u| \leqslant \varepsilon \sigma \sqrt{N}} \epsilon^{-i u v} \psi_{r, t}(u) d u \\
& I_{3}=\int_{\varepsilon \sigma \sqrt{N}<|u| \leqslant d^{-1} \pi \sigma \sqrt{N}} e^{-i u v} \psi_{r, t}(u) d u, \\
& I_{4}=-\int_{A<|u|} \exp \{-i u v-\sqrt{-2 i u}-E(u, z)+E(0, z)\} \frac{E^{t}(u, z)}{E^{t}(0, z)} d u,
\end{aligned}
$$

положительные постоянные $A$ и $\varepsilon$, как показано при доказательстве леммы 2.5 .1 в [5], можно выбрать так, что интегралы $I_{2}, I_{3}, I_{4}$ при достаточно больших $N, n$ становятся сколь угодно малыми. Отсюда и из (12) следует утверждение леммы 5.

Аналогично лемме 2.2.6 из книги [5] доказывается следующее утверждение.

Лемма 6. Пусть $n, N \rightarrow \infty$ так, что $n / N^{2} \rightarrow \infty, r$ кратно $d u r=z N^{2}$, где $z-$ положительная постоянная, $\lambda=1$. Тогда

$$
N P_{r} \rightarrow E(0, z)
$$

Лемма 7. Пусть $n, N \rightarrow \infty$ так, что $n / N^{2} \rightarrow \infty, r$ кратно $d u r=z N^{2}$, где $z$ фиксированное положительное число. Тогда

$$
\mathbf{P}\left\{\nu_{r, 1}=N+n\right\}=\frac{N d \sqrt{z}}{2 n^{3 / 2}}(1+o(1)) \text {. }
$$

Доказателъство. По формуле полной вероятности

$$
\begin{aligned}
\mathbf{P}\left\{\nu_{r, 1}=N+\right. & n\} \\
& =\sum_{0 \leqslant k<n-z N^{2}} \mathbf{P}\left\{\nu_{r}^{(1)}+\ldots+\nu_{r}^{(N-1)}=N-1+k\right\} \mathbf{P}\left\{\tilde{\nu}_{r}^{(N)}=1+n-k\right\} .
\end{aligned}
$$

Положим

$$
\gamma=\left(N^{2} / n\right)^{1 / 7}
$$


разобьем область суммирования на три части:

$$
\begin{aligned}
& M_{1}=\{k: 0 \leqslant k \leqslant \gamma n\}, \\
& M_{2}=\{k: \gamma n<k \leqslant n-\gamma n\}, \\
& M_{3}=\left\{k: n-\gamma n<k<n-z N^{2}\right\}
\end{aligned}
$$

и обозначим соответствующие суммы через $S_{1}, S_{2}, S_{3}$. Тогда

$$
\mathbf{P}\left\{\nu_{r, 1}=N+n\right\}=S_{1}+S_{2}+S_{3} .
$$

Используя лемму 1.3.12 из [5], легко показать, что

$$
S_{1}=\frac{d(1+o(1))}{P_{r} n^{3 / 2} \sqrt{2 \pi B}} \sum_{0 \leqslant k \leqslant \gamma n} \mathbf{P}\left\{\nu_{r}^{(1)}+\ldots+\nu_{r}^{(N-1)}=N+k-1\right\} .
$$

Обозначим $\varphi_{r}(u)$ характеристическую функцию суммы

$$
\nu_{r}^{(1)}+\ldots+\nu_{r}^{(N-1)} .
$$

Тогда аналогично нахождению характеристической функции $\psi_{r}(u)$ в доказательстве леммы 5 , нетрудно показать,что $\varphi_{r}(u)$ сходится к характеристической функции распределения с плотностью

$$
g(u)=\frac{1}{\sqrt{2 \pi}} e^{E(0, z)} \sum_{k=0}^{\infty} \frac{(-1)^{k}}{k !} I_{k}(z, v)
$$

Отсюда и из (14) следует, что

$$
S_{1}=\frac{N d(1+o(1))}{E(0, z) n^{3 / 2} \sqrt{2 \pi B}}
$$

Используя леммы 1.3.12 и 2.3.4 из [5], и лемму 6, легко показать, что

$$
S_{2} \leqslant C_{1} \frac{N^{2}}{\gamma^{3} n^{2}}=o\left(N / n^{3 / 2}\right)
$$

здесь и далее $C_{1}, C_{2}, \ldots$ означают некоторые положительные постоянные. По лемме 1.3.12 из [5]

$$
S_{3} \leqslant \frac{C_{2}}{N^{2}} \sum_{n(1-\gamma)<k<n-z N^{2}} \mathbf{P}\left\{\nu_{r}^{(1)}+\ldots+\nu_{r}^{(N-1)}=N+k-1\right\} .
$$

Легко видеть, что если $n / N^{3} \rightarrow \infty$, то

$$
\mathbf{P}\left\{\nu_{r}^{(1)}+\ldots+\nu_{r}^{(N-1)}=N+k-1\right\}=0 .
$$

Пусть $n / N^{3} \leqslant C_{3}<\infty$. Рассмотрим вероятность

$$
Q_{N-1}=\mathbf{P}\left\{\nu_{n-z N^{2}}^{(1)}+\ldots+\nu_{n-z N^{2}}^{(N-1)}=N+k-1, \nu_{n-z N^{2}}^{(i)} \leqslant z N^{2}, i=1, \ldots, N-1\right\} .
$$


Нетрудно показать, что

$$
Q_{N-1}=\left(\frac{1-P_{r}}{1-P_{n-z N^{2}}}\right)^{N-1} \mathbf{P}\left\{\nu_{r}^{(1)}+\ldots+\nu_{r}^{(N-1)}=N+k-1\right\} .
$$

Согласно лемме 2.2.7 из [5]

$$
\left(1-P_{n-z N^{2}}\right)^{N-1}=1+o(1)
$$

а из леммы 6 следует, что

$$
\left(1-P_{r}\right)^{N-1} \rightarrow e^{-E(0, z)} .
$$

Поэтому из (18) получаєм, что

$$
\mathbf{P}\left\{\nu_{r}^{(1)}+\ldots+\nu_{r}^{(N-1)}=N+k-1\right\}=Q_{N-1} e^{E(0, z)}(1+o(1)) .
$$

Оценим вероятность

$$
Q_{N-1}=\mathbf{P}\left\{\nu_{n-z N^{2}}^{(1)}+\ldots+\nu_{n-z N^{2}}^{(N-1)}=N+k-1, \nu_{n-z N^{2}}^{(i)}, \leqslant z N^{2}, i=1, \ldots, N-1\right\} .
$$

Положим

$$
R(\omega)=\sum_{k \leqslant z N^{2} / d} \exp \{\omega(k d+1)\} \mathbf{P}\left\{\nu_{n-z N^{2}}^{(1)}=k d+1\right\}
$$

Введем независимые одинаково распределенные случайные величины $\nu_{i}(z)$, для которых

$$
\mathbf{P}\left\{\nu_{1}(z)=s d+1\right\}=\exp \left\{(s d+1) /\left(z N^{2}\right)\right\} \frac{\mathbf{P}\left\{\nu_{n-z N^{2}}^{(1)}=s d+1\right\}}{R\left(1 /\left(z N^{2}\right)\right.}
$$

при этом $s \leqslant z N^{2} / d$. Положим

$$
\zeta_{N-1}(z)=\nu_{1}(z)+\ldots+\nu_{N-1}(z)
$$

Нетрудно показать, что

$$
Q_{N-1}=\frac{P\left\{\zeta_{N-1}(z)=N+k-1\right\} R\left(1 /\left(z N^{2}\right)\right)}{\exp \left\{(N+k-1) /\left(z N^{2}\right)\right\}} .
$$

По формуле обращения

$$
\begin{aligned}
\mathbf{P}\left\{\zeta_{N-1}(z)=\right. & N+k-1\} \\
& =\frac{d B}{2 \pi N^{2}} \int_{-\pi N^{2} /(d B)}^{\pi N^{2} /(d B)} \exp \left\{-\frac{i B y}{N^{2}}(N+k-1)\right\} \varphi_{\nu_{1}(z)}^{N-1}\left(B y / N^{2}\right) d y
\end{aligned}
$$

где $\varphi_{\nu_{1}(z)}(u)$ означает характеристическую функцию случайной величины $\nu_{1}(z)$. Рассмотрим $\left|\varphi_{\nu_{1}(z)}(u)\right|$. Нетрудно показать, что

$$
\left|\varphi_{\nu_{1}(z)}(u)\right|^{N-1}=\left|R\left(i u+1 /\left(z N^{2}\right)\right) / R\left(1 /\left(z N^{2}\right)\right)\right|^{N-1} .
$$


Если $k \leqslant z N^{2} / d$, то справедливо неравенство

$$
\exp \left\{(k d+1) /\left(z N^{2}\right)\right\} \leqslant 1+2(k d+1) /\left(z N^{2}\right) .
$$

Отсюда и из соотношений

$$
\begin{gathered}
\frac{2}{z N^{2}} \sum_{k \leqslant z N^{2} / d}(k d+1) \mathbf{P}\left\{\nu_{n-z N^{2}}^{(1)}=k d+1\right\} \leqslant \frac{C_{4}}{z N^{2}} \sqrt{z N^{2} / d}=o\left(\frac{1}{N}\right) \\
\sum_{k>z N^{2} / d} \mathbf{P}\left\{\nu_{n-z N^{2}}^{(1)}=k d+1\right\} \leqslant \frac{C_{5}}{\sqrt{z N^{2} / d}}=o\left(\frac{1}{N}\right)
\end{gathered}
$$

следует, что

$$
\left|R\left(1 /\left(z N^{2}\right)+i u\right)\right| \leqslant\left|\varphi_{\nu_{n-z N^{2}}^{(1)}}(u)\right|+o(1 / N)
$$

где $\varphi_{\nu_{n-z N^{2}}^{(1)}}(u)$ - характеристическая функция случайной величины $\nu_{n-z N^{2}}^{(1)}$. Для любого $u$ справедливо неравенство $\left|\varphi_{\nu^{(1)}}(u)\right| \geqslant C_{6}$, поэтому

$$
\left|\varphi_{\nu_{n-z N^{2}}^{(1)}}(u)\right| \geqslant C_{7} .
$$

Аналогично можно показать, что

$$
R\left(1 /\left(z N^{2}\right)\right)=1+o\left(1 /\left(z N^{2}\right)\right) .
$$

Тогда из (24) и (25) следует, что

$$
\left|\varphi_{\nu(1)}(u)\right|^{N-1} \leqslant C_{8}\left|\varphi_{\nu_{n-z N^{2}}^{(1)}}(u)\right|^{N-1} .
$$

Если $|u| \leqslant \varepsilon N^{2}$, то

$$
\left|\varphi\left(u / N^{2}\right)\right| \leqslant \exp \left\{-\sqrt{|u| / 2 B N^{2}}\right\},
$$

а для $\varepsilon<|u| \leqslant \pi / d$ справедливо неравенство $|\varphi(u)| \leqslant e^{-C_{9}}$. Тогда при $|u| \leqslant \varepsilon N^{2}$

$$
\left|\varphi_{\nu_{n-z N^{2}}^{(1)}}\left(B u / N^{2}\right)\right|^{N-1} \leqslant C_{10} \exp \{-\sqrt{|u| / 2}\},
$$

а при $\varepsilon N^{2}<|u|<\pi N^{2} /(d B)$

$$
\left|\varphi_{\nu_{n-x N^{2}}^{(1)}}\left(B u / N^{2}\right)\right|^{N-1} \leqslant C_{11} e^{-C_{12} N} .
$$

Отсюда, из (23) и (26) следует, что

$$
\mathbf{P}\left\{\zeta_{N-1}(z)=N+k-1\right\} \leqslant C_{13} / N^{2} .
$$

Теперь из (22) получаем, что

$$
Q_{N-1} \leqslant \frac{C_{14}}{N^{2}} \exp \left\{-(N+k-1) /\left(z N^{2}\right)\right\} .
$$

Отсюда и из (19) следует, что

$$
\mathbf{P}\left\{\nu_{r}^{(1)}+\ldots+\nu_{r}^{(N-1)}=N+k-1\right\} \leqslant \frac{C_{15}}{N^{2}} \exp \left\{-(N+k-1) /\left(z N^{2}\right)\right\}
$$


поэтому из (17) получаем, что

$$
\begin{aligned}
S_{3} & \leqslant \frac{C_{16}}{N^{4}} \sum_{n(1-\gamma)<k<n-z N^{2}} \exp \left\{-(N+k-1) /\left(z N^{2}\right)\right\} \\
& \leqslant \frac{C_{17}}{N^{4}} \exp \left\{-n / N^{2}\right\} n \gamma=o\left(N / n^{3 / 2}\right) .
\end{aligned}
$$

Утверждение леммы 7 следует теперь из (13), (15) и (16).

Лемма 8. Пусть $n, N \rightarrow \infty$ так, что $n / N^{2} \rightarrow \infty, r$ кратно $d u r=z N^{2}$, где $z-$ фиксированное положстельное число. Тогда

$$
n^{3 / 2} \mathbf{P}\left\{\nu_{r, 0}=N+n\right\} / N \rightarrow 0 .
$$

Доказателзство. Аналогично тому, как это делалось в доказательстве леммы 7 , легко показать, что если $n / N^{3} \rightarrow \infty$, то $\mathbf{P}\left\{\nu_{r, 0}=N+n\right\}=0$. В случае $n / N^{3} \leqslant$ $C_{18}<\infty$, рассмотрим вероятность

$$
Q_{N}=\mathbf{P}\left\{\nu_{n-z N^{2}}^{(1)}+\ldots+\nu_{n-z N^{2}}^{(N)}=N+n, \nu_{n-z N^{2}}^{(i)} \leqslant z N^{2}, i=1,2, \ldots, N\right\} .
$$

Нетрудно показать, что

$$
\begin{aligned}
Q_{N} & =\left(\frac{1-P_{r}}{1-P_{n-z N^{2}}}\right)^{N} \mathbf{P}\left\{\nu_{r}^{(1)}+\ldots+\nu_{r}^{(N)}=N+n\right\} \\
& =e^{-E(0, z)} \mathbf{P}\left\{\nu_{r}^{(1)}+\ldots+\nu_{r}^{(N)}=N+n\right\}(1+o(1)) .
\end{aligned}
$$

Введем независимые одинаково распределенные случайные величины $\nu_{i}(z), i=$ $1, \ldots, N$, с распределением (21) и пусть

$$
\zeta_{N}(z)=\nu_{1}(z)+\ldots+\nu_{N}(z) \text {. }
$$

Нетрудно показать, что

$$
Q_{N}=\frac{\mathbf{P}\left\{\zeta_{N}(z)=N+n\right\} R\left(1 /\left(z N^{2}\right)\right)}{\exp \left\{(N+n) /\left(z N^{2}\right)\right\}},
$$

где $R(\omega)$ определено соотношением (20). По формуле обращения

$$
\mathbf{P}\left\{\zeta_{N}(z)=N+n\right\}=\frac{d B}{2 \pi} \int^{2} \int_{-\pi N^{2} /(d B)}^{\pi N^{2} /(d B)} \exp \left\{-\frac{i B y}{N^{2}}(N+k-1)\right\} \varphi_{\nu_{1}(z)}^{N}\left(B y / N^{2}\right) d y
$$

Аналогично (27) можно получить оценку

$$
\mathbf{P}\left\{\zeta_{N}(z)=N+n\right\} \leqslant C_{19} / N^{2} .
$$

Отсюда, из (28) и (29) следует, что

$$
\mathbf{P}\left\{\nu_{r}^{(1)}+\ldots+\nu_{r}^{(N)}=N+n\right\} \leqslant e^{E(0, z)} \frac{C_{20}}{N^{2}} \exp \left\{-\frac{N+n}{z N^{2}}\right\}=o\left(N / n^{3 / 2}\right),
$$

что и доказывает лемму 8 . 
Докажем теперь теоремы 3-7. Если выполнены условия теоремы 3, то согласно лемме 2.2.2 из книги [5]

$$
N P_{r-d} \rightarrow \infty, \quad N P_{r+k} \rightarrow \gamma, \quad k=0, d, 2 d, \ldots, s-d .
$$

Поэтому для любого фиксированного $i=0,1, \ldots, j$

$$
\left(1-P_{r-d}\right)^{N-i} \rightarrow 0,\left(1-P_{r+k}\right)^{N-i} \rightarrow e^{-\gamma} .
$$

В [5] показано, что

$$
P_{r+s}=\mathbf{P}\left\{\nu^{(1)}=r+s+l+1\right\}(1+o(1))
$$

и

$$
N \mathbf{P}\left\{\nu^{(1)}=r+s+l+1\right\} \rightarrow 0
$$

Поэтому

$$
\left(1-P_{r+s}\right)^{N-i} \rightarrow 1 \text {. }
$$

Отсюда, из лемм 2,4 , леммы 2.3.2 книги [5] и соотношений $(30),(31)$ следует , что

$$
\mathbf{P}\left\{\nu_{(N-j)}(\mathscr{F}) \leqslant r-d+1\right\} \rightarrow 0
$$

и

$$
\mathbf{P}\left\{\nu_{(N-j)}(\mathscr{F}) \leqslant r+k+1\right\} \rightarrow \sum_{i=0}^{j} \frac{\gamma^{i}}{i !} e^{-\gamma},
$$

что и доказывает теорему 3.

Если выполнены условия теоремы 4, то согласно лемме 2.2.3 из [5], для фиксированных целых $k$

$$
N P_{r+k d} \rightarrow \gamma \alpha^{k+1}(1-\alpha)^{-1}
$$

Поэтому для любого фиксированного $i=0,1, \ldots, j$

$$
\left(1-P_{r+k d}\right)^{N-i} \rightarrow \exp \left\{-\gamma \alpha^{k+1}(1-\alpha)^{-1}\right\} .
$$

Отсюда и из лемм 2, 4 и леммы 2.3.2 из [5] следует теорема 4.

Пусть выполнены условия теоремы 5. Согласно лемме 2.2.5 из [5]

$$
N P_{r} \rightarrow e^{-z}
$$

Поэтому

$$
\left(1-P_{r}\right)^{N-i} \rightarrow e^{-e^{-z}}
$$

Отсюда и из лемм 2,4 и леммы 2.3.2 из [5] получаем утверждение теоремы 5 .

Если выполнены условия теоремы 6, то из леммы 2.2.6 книги [5] следует, что

$$
N P_{r} \rightarrow E(0, z)
$$

Поэтому для любого фиксированного $i=0,1, \ldots, j$

$$
\left(1-P_{r}\right)^{N-i} \rightarrow e^{-E(0, z)} \text {. }
$$

Отсюда и из лемм 2, 5 и леммы 2.3 .2 из [4] получаем теорему 6.

Теорема 7 следует из лемм 6-8 и леммы 2.3 .4 из [4]. 


\section{Список литературы}

1. Meir A., Moon J. W. On the altitude of nodes in random trees. Canad. J. Math. (1978) 30, 997-1015.

2. Ватутин В. А. Ветвящиеся процессы с финальными типами частиц и случайные деревья. Теория вероятностей и ее применения (1994) 39, №4, 699-716.

3. Дрмота М. Распределения высоты листьев корневых деревьев. Дискретная матемаmuxa (1994) 6, №1, 67-82.

4. Колчин В. Ф. Случайные отображения. Наука, Москва, 1984.

5. Павлов Ю. Л. Случайные леса. Карельский научный центр РАН, Петрозаводск, 1996.

6. Бритиков В. Е. Предельные теоремы для максимального объема дерева в случайном лесе из некорневых деревьев. Вероятностные задачи дискретной математики. МИЭМ, Москва, 1987, 84-91.

7. Luczak T., Pittel B. Components of random forests. Combinatorics, Probability and Computing (1992) 1, 35-52.

Статья поступила 22.08.1997. 